\title{
BMJ Open How to adapt existing evidence-based clinical practice guidelines: a case example with smoking cessation guidelines in Canada
}

\author{
Peter Selby, ${ }^{1,2,3,4}$ Katie Hunter, ${ }^{1,5}$ Jess Rogers, ${ }^{5}$ Kelly Lang-Robertson, ${ }^{5}$ \\ Sophie Soklaridis, ${ }^{3,6}$ Virginia Chow, ${ }^{1}$ Michèle Tremblay, ${ }^{7}$ Denise Koubanioudakis, ${ }^{7}$ \\ Rosa Dragonetti, ${ }^{1}$ Sarwar Hussain, ${ }^{1}$ Laurie Zawertailo ${ }^{1,8}$
}

To cite: Selby P, Hunter K, Rogers J, et al. How to adapt existing evidence-based clinical practice guidelines: a case example with smoking cessation guidelines in Canada. BMJ Open 2017;7:e016124. doi:10.1136/ bmjopen-2017-016124

\section{- Prepublication history and} additional material for this paper are available online. To view these files, please visit the journal (http://dx.doi.org/10. 1136/bmjopen-2017-016124).

Received 26 January 2017 Revised 30 August 2017 Accepted 6 October 2017
CrossMark

For numbered affiliations see end of article.

Correspondence to

Dr Peter Selby;

peter.selby@camh.ca

\section{ABSTRACT}

Objective To develop and encourage the adoption of clinical practice guidelines (CPGs) for smoking cessation in Canada by engaging stakeholders in the adaptation of existing high-quality CPGs using principles of the ADAPTE framework.

Methods An independent expert body in guideline review conducted a review and identified six existing CPGs, which met a priori criteria for quality and potential applicability to the local context. Summary statements were extracted and assigned a grade of recommendation and level of evidence by a second expert panel. Regional knowledge exchange brokers recruited additional stakeholders to build a multidisciplinary network of over 800 clinicians, researchers and decision-makers from across Canada. This interprofessional network and other stakeholders were offered various opportunities to provide input on the guideline both online and in person. We actively encouraged end-user input into the development and adaptation of the guidelines to ensure applicability to various practice settings and to promote adoption. Results The final guideline contained 24 summary statements along with supporting clinical considerations, across six topic area sections. The guideline was adopted by various provincial/territorial and national government and non-governmental organisations.

Conclusions This method can be applied in other jurisdictions to adapt existing high-quality smoking cessation CPGs to the local context and to facilitate subsequent adoption by various stakeholders.

\section{BACKGROUND}

Smoking cessation interventions are one of the most cost-effective interventions in medicine. ${ }^{1}$ Even brief simple advice by healthcare providers (HCPs) produce small but clinically significant increases in quit rate ${ }^{23}$ that are associated with important public health benefits at a population level. ${ }^{4}$ More intensive interventions confer even greater benefits. ${ }^{3}$ Historically, a large proportion of smokers in Canada who wanted to make a quit attempt have not consistently received help to quit

\section{Strengths and limitations of this study}

- This paper describes the methodology used to develop clinical practice guidelines for smoking cessation in Canada by adapting existing highquality guidelines using the ADAPTE framework and engaging key stakeholders and end-users in the process. This method can be applied in other jurisdictions to adapt existing high-quality guidelines to the local context to facilitate subsequent adoption.

- The online wiki platform designed to foster engagement and allow for discussion of the guideline by network members was used minimally. In-person strategies were more successful for gathering feedback from end-users to inform development of the guideline.

- Patient-level outcome data were not available to evaluate the impact of development and dissemination of the guideline.

from their HCPs. In 2005, $>88 \%$ of the close to 5 million Canadians who smoked made at least one visit to a healthcare provider in the previous year, yet only about half were advised to reduce or quit smoking and even fewer received information regarding smoking cessation aids. ${ }^{5}$

Although clinical practice guidelines (CPGs) for smoking cessation have long recommended that HCPs assess current smoking status and provide assistance with smoking cessation, ${ }^{3}$ there has been poor uptake of these recommendations. Commonly cited barriers to guideline implementation or uptake are attitudinal barriers such as acceptance of guidelines, willingness to change and the day-to-day experiences of practitioners not reflected in the guideline. ${ }^{6}$ As a result, much research has been done to enhance guideline development methodology to ensure greater consideration of implementation during the development 
process, ${ }^{7-10}$ with the aim to increase the applicability of CPGs to target end-users, resulting in a greater impact on clinical practice.

Article 14 of the WHO Framework Convention on Tobacco Control (WHO FCTC) states that 'each Party shall develop and disseminate appropriate, comprehensive and integrated guidelines based on scientific evidence and best practices, taking into account national circumstances and priorities'. ${ }^{11}$ Canada is a signatory to this framework, but previously did not have a national smoking cessation guideline adapted to the Canadian context. A number of CPGs for smoking cessation existed internationally, ${ }^{3}{ }^{12}$ in addition to high-quality Cochrane reviews. ${ }^{13} 14$ However, no independent high-quality CPG had been previously developed and implemented consistently across Canada. Health Canada released a call for proposals to develop such guidelines in 2006, and CAN-ADAPTT (the Canadian Action Network for the Advancement, Dissemination and Adoption of Practice-informed Tobacco Treatment) was formed in 2008 (http://www.nicotinedependenceclinic.com/English/ CANADAPTT). To prevent duplication of effort and cost, existing guidelines could be adapted but needed specific attention to transcontextual issues. ${ }^{15}$ The unique circumstances in Canada include a publicly funded healthcare system, special populations such as Aboriginal peoples, expanded roles of a variety of HCPs and regional variations in priorities and capacity in clinical prevention. These highlight the need for a guideline specific to the Canadian context. Therefore, our two main goals were to use evidence-based methods to synthesise existing highquality guidelines and to engage end-users to contextualise the recommendations in order to increase adoption and dissemination of the guidelines within Canada.

\section{METHODS}

The development of the guidelines met all the recommended elements described by the Institute of Medicine (IOM) and Guidelines International Network (G-I-N). ${ }^{1617}$ To develop the guideline we used the following three-step process. First, we situated the project in the Knowledge to Action (KTA) Cycle to position the guideline for subsequent implementation. ${ }^{18}$ We then used the ADAPTE framework to guide the transcontextual adaptation. ${ }^{19}$ Within the ADAPTE model, we used the Appraisal of Guidelines for Research and Evaluation (AGREE) Instrument ${ }^{20}$ to rate and select appropriate guidelines. Lastly, we engaged a variety of end-users in the process to facilitate subsequent adoption. ${ }^{21}$ This was facilitated by employing knowledge brokers and establishing a network. These two activities have been identified as promising methodologies to facilitate knowledge exchange. ${ }^{22}$

As the first step, we synthesised existing research as recommended in the 'Knowledge Funnel' component of the KTA model. ${ }^{18}$ This represents the process through which knowledge is distilled to meet the needs of knowledge end-users. Given the existence of several guidelines, we relied on knowledge synthesis of existing guidelines to produce knowledge tools and products instead of rereviewing primary literature. This was done to save time and effort. Although the KTA model describes sources of information such as the Cochrane collaboration, it does not inform how existing CPGs are to be adapted to the local context. We identified the ADAPTE framework as a possible framework to guide the adaptation process since it specifically describes steps to consider transcontextual issues without losing the validity of the evidencebased practice. The ADAPTE process, first described in chemotherapy guidelines, outlines a systematic approach for adapting existing guidelines so they are appropriate for use in a different cultural or organisational context, taking into account the needs, priorities, legislation, policies and resources of the intended setting. ${ }^{19}$ The main steps include: search for existing guidelines; quality assessment of the guidelines; adaptation and applicability to the target setting; literature update; adaptation of the format; external review and finally adoption, endorsement and implementation. The ADAPTE framework involves the engagement of end-users in the guideline adaptation process to provide context for implementation and enhance uptake of the final guideline. ${ }^{15} 1923$ The core principles on which the ADAPTE process are based include: respect for evidence-based principles in guideline development; ensuring guideline quality through use of reliable and consistent methods; involvement of key stakeholders to promote acceptance and use of the guideline; consideration of context to ensure applicability to local practice; flexibility of format to accommodate specific needs and acknowledgement of source materials. ${ }^{15}$

\section{Synthesis of the evidence base}

We first identified and evaluated existing guidelines in smoking cessation. A preliminary review of published and grey literature revealed several existing CPGs for smoking cessation from a variety of jurisdictions and one pharmaceutical company sponsored guideline from Canada.

\section{Identification of existing clinical practice guidelines: two cycles}

To establish a strong evidence base from which CAN-ADAPTT would build its Canadian practice-informed guideline, the Guidelines Advisory Committee (GAC), an independent, not-for-profit organisation was engaged to identify and assess existing CPGs. They provided independence to the process and mitigated potential bias by experts in smoking cessation with ties to commercial interests in smoking cessation who formed part of the Guideline Development Group (GDG, see below). The GAC comprises leaders in guideline development and evaluation methods with a trained reviewer pool of practising family physicians. Family physicians are the key primary care providers in Canada representing the primary target audience. The first iteration was conducted in November 2006. The GAC used a previously established process ${ }^{24}$ to conduct a full review of existing 
CPGs in smoking cessation published between 2002 and 2006.

The search was conducted using subject headings and focused terms such as smoking, tobacco or nicotine, in Ovid MEDLINE, Ovid Embase, guideline repositories such as National Guideline Clearinghouse, renowned developers with a history of developing high-quality guidelines such as the National Institute for Health and Clinical Excellence and websites of national and international specialty societies. A general Internet search was also conducted to ensure that no significant guidelines were missed. The search was limited to guidelines published in English. At that time, nine guidelines were identified (see box 1), which met basic predetermined quality criteria (ie, contained recommendations linked to the evidence and included some indication that a systematic search of the literature had been conducted). The second search of the published literature was conducted in January 2009 using an identical search and data extraction strategy. Five additional guidelines published since the previous search in 2006 met inclusion criteria (see box 1).

\section{Appraisal of existing guidelines}

The 14 guidelines identified in both reviews were evaluated for quality by four independent GAC reviewers using the AGREE instrument (see online supplementary table 1).$^{20}$ Reviewers were practising family physicians in Ontario who had each been formally trained in the application of the AGREE instrument by the Centre for Effective Practice. The AGREE instrument was developed in 2001 as a tool to assess guideline quality and is an internationally recognised and validated tool. The AGREE instrument includes 21 items each scored on a scale from 1 to 4, categorised under six domains: scope and purpose, stakeholder involvement, rigour of development, clarity and presentation, applicability and editorial independence. The domain scores for all appraisers are combined and standardised as a total percentage of the maximum possible score for that domain. Guidelines are also assigned a global assessment of 'strongly recommend', 'recommend' or 'not recommend'. Along with the AGREE instrument, reviewers were also asked to answer eight additional questions developed by CAN-ADAPTT to help capture information on the applicability of the recommendations to the Canadian context (see box 2). This was done to explicitly identify issues of applicability to facilitate adoption of the Canadian guideline.

The AGREE instrument does not provide thresholds to indicate whether a guideline is of high-quality, but instead requires the appraiser to make an overall assessment taking each of the appraisal criteria into consideration. ${ }^{20}$ The GAC used the following criteria to identify high-quality guidelines for the purposes of this project: received an overall AGREE rating of 'strongly recommend' from at least three of the four reviewers; had a combined score of over $75 \%$ in the domain for rigour of development as well as at least three other domains and were determined to be applicable in the Canadian

\section{Box 1 Existing clinical practice guidelines identified}

\section{2-2006}

- National Advisory Committee on Health and Disability (National Health Committee). (May 2002). Guidelines for smoking cessation. Retrieved 24 October 2007 from: http://www.nzgg.org.nz/ guidelines/0025/Smoking_Cessation_full.pdf

- Institute for Clinical Systems Improvement (ICSI). (June 2004). Healthcare guideline: tobacco use prevention and cessation for adults and mature adolescents. Retrieved 24 October 2007 from: http://www.icsi.org/tobacco_use_prevention_and_cessation_for adults/tobacco_use_prevention_and_cessation_for_adults_and mature_adolescents_2510.html*

- Institute for Clinical Systems Improvement (ICSI). (June 2004). Tobacco use prevention and cessation for infants, children and adolescents. Retrieved 24 0ctober 2007 from: http://www.icsi.org/ tobacco_use_prevention_and_cessation_for_children/tobacco_ use_prevention_and_cessation_for_infants__children_and_ adolescentsn_and_adolescents_full_version_.html ${ }^{*}$

- Registered Nurses' Association of Ontario (RNA0). (October 2003). Integrating smoking cessation into daily nursing practice. Retrieved 26 October 2007 from: http://www.rnao.org/bestpractices/PDF/ BPG_smoking_cessation.pdf*

- Management of Tobacco Use Working Group. (June 2004). VA/ $\mathrm{DoD}$ clinical practice guideline for the management of tobacco use. Version 2.0a. Washington (DC): Department of Veteran Affairs, Department of Defense. Retrieved 24 October 2007 from: http:// www.oqp.med.va.gov/cpg/TUC3/G/TUC-2004.pdf

- National Institute for Health and Clinical Excellence. (March 2006). Brief interventions and referral for smoking cessation in primary care and other settings. Public Health Intervention Guidance No 1. Retrieved 24 October 2007 from: http://www.nice.org.uk/download. aspx?0=299608

- Le Foll B, Melihan-Cheinin P, Rostoker G, et al. for the working group of AFSSAPS. (2005). Smoking cessation guidelines: Evidence-based recommendations of the French Health Products Safety Agency. European Psychiatry: the Journal of the Association of European Psychiatrists, 20(5-6), 431-441.

- Smoking cessation guidelines for Australian general practice. (2004). Retrieved 24 October 2007 from: http://www.health.gov.au/internet/ wcms/publishing.nst/content/health-pubhlth- publicat-documentsmoking_cessation-cnt.htm/\$FILE/smoking_cessation.pdf

- EBM Guidelines. (December 2006). Smoking cessation.

\section{6-2008}

- US Department of Health and Human Services Public Health Service. (2008, May). Clinical practice guideline: Treating tobacco use and dependence: 2008 update.*

- Reichert J, de Araújo AJ, Gonçalves,CM, et al. (2008, October). Smoking cessation guidelines: 2008. Jornal Brasileiro de Pneumologia, 34(10), 845-880.

- Ministry of Health. (2007 August). New Zealand smoking cessation guidelines. Wellington: Ministry of Health.*

- Registered Nurses' Association of Ontario (RNA0). (March 2007). Integrating smoking cessation into daily nursing practice.*

- Tønnesen P, Carrozzi L, Fagerstrom,KO, et al. (2007). Smoking cessation in patients with respiratory diseases: a high priority, integral component of therapy. European Respiratory Society Task Force. European Respiratory Journal, 29, 390-417.

*High-quality guidelines identified as the evidence base for the Canadian guideline. 
Box 2 Additional questions to address applicability to the Canadian context

1. The recommendations made in the guideline are appropriate for the intended users (ie, you and your colleagues) to perform.

2. The recommendations made in the guideline are practical for the intended users (ie, you and your colleagues) to perform.

3. The recommendations made in the guideline are consistent with patient treatment expectations.

4. The recommendations are compatible with existing attitudes and beliefs of the guideline's intended users (ie, you and your colleagues).

5. The recommendations can be performed by the guideline's intended users (ie, you and your colleagues) without the acquisition of new competencies (knowledge, skills, etc).

6. What new competencies, if any, would be required?

7. Criteria can be extracted from the guideline that would permit the measurement of specific outcomes related to the recommendations.

8. Criteria can be extracted from the guideline that would permit the measurement of adherence to the recommendations.

context. The rigour of development domain includes questions relating to the systematic approach and transparency of methods as well as the extent to which recommendations are linked to the evidence. This domain was identified by the GAC as critical to determining the quality of a CPG; for this reason, guidelines that did not score at least $75 \%$ in this domain were not considered high-quality and were not considered for use when developing the guideline. These criteria were based on the GAC's expertise and years of experience reviewing and endorsing guidelines. Applicability to the Canadian context was based on scores of the AGREE 'applicability' domain, as well as results from the CAN-ADAPTT applicability questionnaire. One guideline (the Institute for Clinical Systems Improvement guideline for adults and mature adolescents, see box 1) that received two 'strongly recommend' and two 'recommend' overall ratings also scored over $75 \%$ in five of the six AGREE domains, and was also included for this reason. Ultimately, six highquality guidelines were selected for use in developing the Canadian clinical practice guideline (see figure 1 for a detailed process diagram and box 1 for the list of highquality guidelines selected).

\section{Role of the guideline development group}

The CAN-ADAPTT coordinating team invited a variety of HCPs from across Canada to adapt the recommendations to develop a practice-informed CPG. There were seven members of the GDG including a family physician, public health researcher, health psychologist and physician specialists from respirology, addiction and cardiology. GDG members were identified by the Chair of the GDG to include experts in each guideline topic area while ensuring a multidisciplinary and nationally representative committee. Representatives from four provinces (Ontario, Nova Scotia, Alberta and Quebec) were included for regional representation. Each GDG member received a modest honorarium to support their attendance at guideline development meetings and reimbursement for travel and accommodation for in-person meetings. Seven GDG meetings were held from 2006 to 2010. Each GDG member confirmed that they had not received funds from the Tobacco Industry. All network members and GDG Section Leads had to disclose any potential or perceived conflicts of interest, especially with pharmaceutical companies. Members of the GDG without commercial conflicts were asked to be Section Leads and adjudicate the final recommendations. In addition, we ensured that having a majority without conflicts in each section subgroup diluted the effects of any potential commercial bias. Lastly, we did not specify any particular pharmacotherapy in the first iteration of the guideline given that each medication (nicotine replacement, bupropion and varenicline) required a primary review of the extant literature by Canadian prescribers and was beyond the scope of the first iteration of the CAN-ADAPTT guideline process. Taken together, we believe the independence of the GAC and the steps taken to dilute any effects of commercial bias led to a balanced set of recommendations. Given the large number of potential guideline target end-users, representation on the GDG from all end-users was not feasible. However, end-users were involved throughout the development process (described below) to ensure the guideline reflected the experiences and needs of as many HCPs as feasible with the funds available.

\section{Attributing level of evidence and grade of recommendation}

Summary statements contained in CAN-ADAPTT's guideline ${ }^{25}$ were based on the recommendations extracted from the six high-quality guidelines identified, along with input from GDG members. The six high-quality guidelines that were chosen each had different classifications for the grade of recommendation and level of evidence. The GRADE (Grading of Recommendations, Assessment, Development and Evaluation) Working Group's guide for grading strength of recommendations and quality of evidence $^{26-28}$ was used as a tool to bring CAN-ADAPTT's GDG to consensus on the level of evidence and grade of recommendation for each of the summary statements they developed. Each summary statement was attributed a level of evidence (A, B or C) and a grade of recommendation ( 1 =strong or $2=$ weak). This is a transparent and reproducible method for grading recommendations and could also be used in subsequent iterations of the guideline if primary studies were evaluated.

\section{End-user adaptation}

Many internationally developed guidelines have relied primarily on the expertise of subject matter experts. ${ }^{3} 1229$ In contrast, to complement the contributions from the GDG, the CAN-ADAPTT coordinating team and knowledge exchange brokers actively engaged target end-users throughout the development process. To meet the needs 
CAN ADAPTT

Developing Canadian Clinical Practice Guidelines for Smoking Cessation

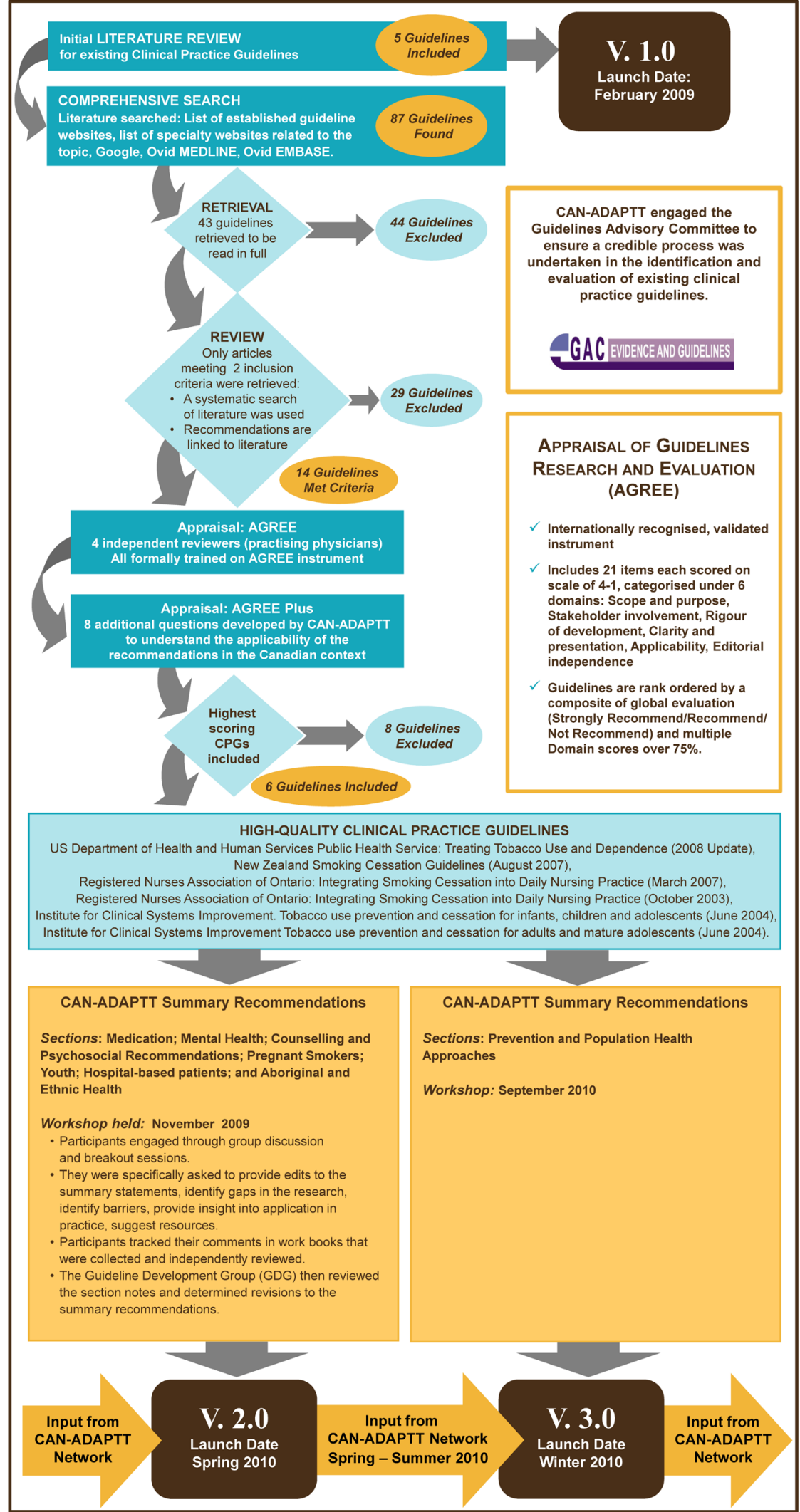

Figure 1 Methodology used for identification of existing high-quality clinical practice guidelines. 
of Francophone communities in Quebec and across Canada, the complete CPG was translated into French. It was then reviewed by experts in smoking cessation in Quebec, including two public health experts and a specialist physician with extensive clinical experience in smoking cessation interventions. They confirmed the guideline was applicable to Francophone communities, relevant to clinical practice and recommended its dissemination in Quebec and among French-speaking HCPs working in Canada (www.nicotinedependenceclinic. com/Francais/CANADAPTT).

\section{CAN-ADAPTT network}

An interprofessional network of over 800 members across Canada was established to provide an opportunity for end-users to refine and adapt the guideline, as well as contribute to the dissemination and implementation efforts. A number of strategies were used to promote CAN-ADAPTT and recruit members. This included invitations to join the network that were sent to over 100 professional associations and organisations representing various related disciplines, presentations and displays at practitioner conferences, email blasts and other print promotional materials, conducting workshops for HCPs and publishing articles in professional journals and newsletters. Network members were recruited from across Canada, and almost all Canadian provinces and territories were represented. Approximately, two-thirds of the members were HCPs, with a wide range of disciplines represented, including nurses (25\%), dental assistants/ hygienists (24\%), respiratory therapists or asthma educators $(8 \%)$, counsellors/therapists $(7 \%)$, pharmacists $(7 \%)$, nurse practitioners $(6 \%)$, social workers $(5 \%)$, general practitioner or family physicians $(4 \%)$, specialist physicians $(3 \%)$, dentists $(2 \%)$, psychologists $(2 \%)$, chiropractors $(1 \%)$, occupational therapists $(1 \%)$, dietitians/ nutritionists $(1 \%)$, physiotherapists $(<1 \%)$ and other (3\%). Target end-users of the CPG extended beyond the various HCPs to researchers and policy/decision makers to impress on the need for further research as well as support for implementation and dissemination of the guideline. Network members were from over 400 organisations (see online supplementary table 2).

End-user input from the network was actively sought throughout the development process to help ensure an understanding of the current research and gaps, barriers to practice and relevant clinical questions to inform recommendation development. They were also invited to review the CPG in draft format and provide insight into the clinical considerations and existing resources (clinical tools, websites, etc) contained in the CPG. A number of strategies used in an attempt to engage end-users throughout the guideline development process are described below.

\section{Role of knowledge exchange brokers}

Four individuals acted as regional knowledge exchange brokers (for Atlantic Canada, Ontario, Quebec and
Western Canada) to recruit and attempt to engage practitioner groups and individuals across Canada to join the network and collaborate with the CAN-ADAPTT initiative. This acted to decentralise activity and ensure national representation within the network. HCP involvement and feedback was invited through guideline revision meetings, collaborative workshops, stakeholder meetings and an online discussion board. Each knowledge exchange broker had master's level experience and a background in public health, and were hired following a competitive selection process. Three of the four brokers were situated in Ontario as part of the main team and had access to the internet, telephone and travel budgets to visit their respective regions. Due to the distinct nature of Quebec and the infrastructure of the Institut national de santé publique du Québec, the knowledge broker for Quebec was situated there and was under the direct supervision of a CAN-ADAPTT executive committee member (MT) also situated in Quebec; she also had access to resources and a budget for provincial engagement and dissemination.

\section{Engaging end-users in guideline development}

The CAN-ADAPTT initiative held a face-to-face workshop at annual general meetings (AGMs) in 2009 and 2010. Invitations to the AGMs were sent to all CAN-ADAPTT network members and were also widely circulated to healthcare-related professional associations and other stakeholders across Canada (eg, Newfoundland Smokers Helpline, Ontario Tobacco Research Unit, Manitoba Tobacco Reduction Alliance). These workshops provided an opportunity for guideline revisions and promoted cross-disciplinary discussion by allowing network members and various stakeholders from across Canada to provide feedback on initial versions of the guideline. The format included small group discussion for each of the guideline sections and was facilitated by the respective GDG Section Lead. Each workshop participant was provided with a workbook to capture his or her feedback. In addition, a CAN-ADAPTT staff member participated in each breakout session and was responsible for taking notes, which were then reviewed and approved by each breakout group lead facilitator. Input from the participant workbooks and the notes were compiled and provided to the GDG for consideration in the drafting and revision of the clinical considerations portion of each guideline section.

Between meetings, additional opportunities were provided for CAN-ADAPTT network members to provide input and feedback on the guideline. Regional knowledge exchange brokers met with various regional organisations and associations to seek input on the guideline, discuss potential opportunities to further engage HCPs, researchers and decision makers in the region as well as discuss any other opportunities for collaboration. Collaborative workshops targeted at various HCP groups were held in an effort to educate practitioners about the Canadian guideline, to offer practical advice (often from HCPs within their discipline) on how to implement the 
guideline into practice, and seek feedback on barriers and enablers to implementing the guideline. This input was incorporated into the clinical considerations section of the guideline. Additionally, teleconferences, webinars and online surveys were conducted to further discuss the guideline, strategies for implementation and to gather feedback on the guideline.

\section{Use of an online platform}

By launching the guideline and seeking feedback online throughout the guideline development process, knowledge exchange occurred rapidly. The use of an online discussion board allowed feedback to be provided to the CAN-ADAPTT coordinating team, and among the network members, allowing others to view comments and consider the impact for their own setting. To ensure sustainability of this dynamic, practice-informed guideline, an online wiki platform was launched to host the guideline and allow network members to continuously update specified portions of the guideline. Summary statements are based on high-quality evidence and expert opinion, however network members are able to provide contributions to other sections of the guideline including 'clinical considerations' and 'tools and resources'.

\section{RESULTS}

There were 24 summary statements produced for the guideline (table 1$),{ }^{25}$ along with supporting clinical considerations. These were posted online in the Spring of 2010. The Canadian guideline includes six topic area sections. Although overall prevalence has declined, select subpopulations have not experienced similar declines in smoking and currently bear most of the burden with prevalence rates two to three times that of the general population, and we therefore included these as specific areas for consideration: Aboriginal peoples, ${ }^{30}$ mental illness and/or other addictions ${ }^{31}$ and youth. ${ }^{32}$ Additional populations were identified as being at increased health risk from smoking and were also included: hospital-based populations ${ }^{33}$ and pregnant and breastfeeding women. ${ }^{34}$ Pharmacotherapy was also identified as a topic, however, given the nature of the rapidly changing evidence and policy in this area, a more traditional guideline development process (with involvement limited to prescribers) was undertaken for that section and will be outlined in a separate paper.

Approximately 50-60 participants representing a range of organisations and professions attended each 1-day workshop at the AGM, during which they had the opportunity to provide feedback on the guideline. Feedback was incorporated into 79 clinical considerations, ${ }^{25}$ see table 2 for examples from each guideline section.

Dissemination efforts included the following: the guideline was provided to provincial, territorial and federal government and non-governmental organisations to be integrated into their respective dissemination activities; exhibits and presentations were given about the
Canadian guideline at various provincial and national conferences; the summary statements were incorporated into a book about tobacco reduction and cessation ${ }^{35}$; and the guideline is covered in the curriculum of the internationally accredited Training Enhancement in Applied Cessation Counselling and Health certificate programme, which provides training to HCPs in order to build capacity in evidence-based tobacco dependence treatment. ${ }^{36}$ Adoption of the guideline was evident by several agencies including the: Canadian Cardiovascular Harmonized National Guidelines Endeavour, harmonised guidelines for heart disease, hypertension, lipids and cholesterol ${ }^{37}$; Canadian Stroke Best Practice Recommendations ${ }^{38}$; Canadian Society of Respiratory Therapists ${ }^{39}$ and the Regional Municipality of Peel in Ontario. ${ }^{40}$

The guideline continues to be housed on the Centre for Addiction and Mental Health servers and will be updated as funding allows. Additionally, the guideline will be sustained by various partner organisations and programme that have embedded the information and website link in various materials. Having an online interactive experience allows the guideline to be available at a relatively low cost.

\section{DISCUSSION}

This paper describes the process used to produce a practice-informed, evidence-based smoking cessation CPG for the Canadian context. This method focused on building from existing high-quality guidelines and engaging interprofessional end-users in the process to encourage adoption. Guideline development and implementation are complex activities that require models of knowledge to action as well as methods of implementation. Given there was no need for a de novo guideline, we focused our efforts on contextualisation and potential implementation. ${ }^{41}$ This paper describes one process of adaptation of the evidence base for Canada while engaging interdisciplinary end-users in the production of the guideline to facilitate a practice-informed guideline to enhance chances of adoption. Our assumption was that engaging target end-users at the outset to better understand needs, barriers and gaps would allow us to address those needs in order to minimise the disconnect between knowledge creation and implementation. During the process, we recognised that specific pharmacotherapy recommendations would require a primary review of existing randomised clinical trials and meta-analyses using GRADE. There was concern that the included existing guidelines did not have pharmacotherapy recommendations based on current published literature and so a separate project was initiated in 2011 to address this limitation (not described in detail in this paper). In addition, to help engage Francophones in Quebec, a separate team was involved in adaptation of the guidelines in Quebec to ensure national inclusion.

In undertaking this approach to guideline development, one of the challenges encountered was ensuring an 
Table 1 Canadian smoking cessation guideline summary statements

\section{Summary statements}

GRADE

Counselling and psychosocial approaches

1. ASK: tobacco use status should be updated, for all patients/clients, by all healthcare providers on a regular basis.

2. ADVISE: healthcare providers should clearly advise patients/clients to quit.

3. ASSESS: healthcare providers should assess the willingness of patients/clients to begin treatment to achieve $1 \mathrm{C}$ abstinence (quitting).

4. ASSIST: every tobacco user who expresses the willingness to begin treatment to quit should be offered assistance.

a) Minimal interventions, of 1-3 min, are effective and should be offered to every tobacco user. However, there

is a strong dose-response relationship between the session length and successful treatment, and so intensive interventions should be used whenever possible.

b) Counselling by a variety or combination of delivery formats (self-help, individual, group, helpline, web-based) $1 \mathrm{~A}$ is effective and should be used to assist patients/clients who express a willingness to quit.

c) Because multiple counselling sessions increase the chances of prolonged abstinence, healthcare providers should provide four or more counselling sessions where possible.

d) Combining counselling and smoking cessation medication is more effective than either alone, therefore both $1 \mathrm{~A}$ should be provided to patients/clients trying to stop smoking where feasible.

e) Motivational interviewing is encouraged to support patients/clients willingness to engage in treatment now and $1 \mathrm{~B}$ in the future.

f) Two types of counselling and behavioural therapies yield significantly higher abstinence rates and should be included in smoking cessation treatment: 1) providing practical counselling on problem solving skills or skill training and 2) providing support as a part of treatment.

5. ARRANGE: healthcare providers:

a) should conduct regular follow-up to assess response, provide support and modify treatment as necessary. $1 \mathrm{C}$

b) are encouraged to refer patients/clients to relevant resources as part of the provision of treatment, where appropriate.

Pregnant and breastfeeding women

1. Smoking cessation should be encouraged for all pregnant, breastfeeding and postpartum women.

2. During pregnancy and breastfeeding, counselling is recommended as first-line treatment for smoking cessation. $1 \mathrm{~A}$

3. If counselling is found ineffective, intermittent dosing nicotine replacement therapies (such as lozenges, gum) are $1 \mathrm{C}$ preferred over continuous dosing of the patch after a risk-benefit analysis.

4. Partners, friends and family members should also be offered smoking cessation interventions. $2 B$

5. A smoke-free home environment should be encouraged for pregnant and breastfeeding women to avoid $1 \mathrm{~B}$ exposure to second-hand smoke.

Youth (children and adolescents)

1. Healthcare providers who work with youth (children and adolescents) should obtain information about tobacco $1 \mathrm{~A}$ use (cigarettes, cigarillos, waterpipe, etc) on a regular basis.

2. Healthcare providers are encouraged to provide counselling that supports abstinence from tobacco and/or cessation to youth (children and adolescents) that use tobacco.

3. Healthcare providers in paediatric healthcare settings should counsel parents/guardians about the potential $2 \mathrm{C}^{45}$ harmful effects of second-hand smoke on the health of their children.

Aboriginal peoples*

1. Tobacco misuse $\dagger$ status should be updated for all Aboriginal peoples by all healthcare providers on a regular basis.

2. All healthcare providers should offer assistance to Aboriginal peoples who misuse tobacco with specific emphasis on culturally appropriate methods.

3. All healthcare providers should be familiar with available cessation support services for Aboriginal peoples. 
Table 1 Continued

1. All patients should be made aware of hospital smoke-free policies.

$1 \mathrm{C}$

2. All elective patients who smoke should be directed to resources to assist them to quit smoking prior to hospital $1 \mathrm{~B}$ admission or surgery, where possible.

3. All hospitals should have systems in place to:
a) identify all smokers;
$1 \mathrm{~A}$
b) manage nicotine withdrawal during hospitalisation;
$1 \mathrm{C}$
c) promote attempts towards long-term cessation;
$1 \mathrm{~A}$
d) provide patients with follow-up support post-hospitalisation.

$1 \mathrm{~A}$

4. Pharmacotherapy should be considered:

a) to assist patients to manage nicotine withdrawal in hospital; $1 \mathrm{C}$

b) for use in-hospital and posthospitalisation to promote long-term cessation.

1B

Mental health and/or other addiction(s)

1. Healthcare providers should screen persons with mental illness and/or addictions for tobacco use.

$1 \mathrm{~A}$

2. Healthcare providers should offer counselling and pharmacotherapy treatment to persons who smoke and have $1 \mathrm{~A}$ a mental illness and/or addiction to other substances.

3. While reducing smoking or abstaining (quitting), healthcare providers should monitor the patients'/clients'

$1 \mathrm{~A}$ psychiatric condition(s) (mental health status and/or other addiction(s)). Medication dosage should be monitored and adjusted as necessary.

*Aboriginal peoples is used as an inclusive term, which includes First Nations (both on and off reserve), Inuit and Métis. This is not meant to take away from the diversity that exists among Aboriginal peoples.

†Tobacco misuse does not refer to tobacco use for traditional/ceremonial purposes.

appropriate balance between the evidence base, expert opinion and practitioner feedback was reflected in the guideline. It was determined that in order to appropriately balance evidence and clinical practice, end-users would provide feedback and input to inform the scope and content of the clinical considerations sections, suggest tools and resources to implement the guideline, identify current gaps in knowledge and identify important barriers and opportunities for adoption of the guideline. Summary statements would be based on the best available evidence from existing high-quality guidelines with input from established experts in the area. This approach allowed for a combination of evidence and experience of HCPs to be represented in the guideline.

Various opportunities for end-users to engage in the guideline development process provided different levels of success in collecting input. An online platform was used in an effort to address the commonly cited barrier to uptake of traditionally developed guidelines quickly becoming out of date, which has been reinforced by the description of the need for a 'living guideline' by Davis $e t$ $a l^{42}$. The online platform would allow for rapid updating and dissemination of the guideline, as necessary. However, the use of online platforms as a mechanism to provide feedback on the guideline proved to be a challenge as the number of end-users engaging in the online discussion board was significantly lower than expected. This may be a result of users not being familiar or comfortable with participating in an electronic medium. Appropriate use of these technologies/platforms requires investigation in order to explore the utility of an online and iterative process for guideline development. The most successful activity in terms of gathering feedback from end-users to inform development of the guideline was the workshops at AGMs. This may reflect the preference of end-users to network, collaborate and provide feedback in person rather than online and to participate in structured discussions. The regional knowledge exchange brokers for Western and Atlantic Canada were situated in Ontario due to administrative reasons, incurring high travel costs. This limited the number of personal interactions with end-users in those regions. Contracting with local agencies and consultants as regional brokers could have improved engagement in these provinces as we had done in Quebec.

A recent systematic review of 35 handbooks on CPG development concluded that there are no established standards for updating guidelines and state that $40 \%$ suggest a 2-year to 3-year gap in updating CPGs. ${ }^{43}$ Recommendations are not explicit on updating of the Canadian guidelines as recommended by G-I-N or IOM primarily because the process was conceptualised before the project began. ${ }^{1617}$ However, the guidelines were updated in 2009, prompted by the release of the US guidelines ${ }^{3}$ in 2008 , and a section on pharmacotherapy was updated in $2012^{35}$ due to the release of several primary studies on the use of varenicline and nicotine replacement including headto-head studies. Currently, the pharmacotherapy section is being updated and due for release as an updated decision aid for prescribers in 2017. The other sections do not 
Table 2 Examples of clinical considerations from the Canadian smoking cessation guideline

Section Clinical considerations

Counselling and

Psychosocial Approaches

A systematic approach to asking about tobacco use is best. Documenting tobacco status can involve medical questionnaires, stickers on client charts, electronic health records, chart reminders or through computer reminder systems.

All healthcare providers should be encouraged to obtain training in cessation counselling.

Pregnant and Breastfeeding Evidence from a recent systematic review and meta-analysis demonstrated negative perinatal Women

outcomes (eg, trend towards lower birth weight, smaller head circumference and congenital anomalies) associated with second-hand smoke exposure. Therefore, pregnant and breastfeeding women should avoid this environmental risk.

Including partners, friends and/or family in a pregnant smoker's quit attempt is essential to increase the likelihood of successful smoking cessation interventions.

$\begin{array}{ll}\text { Youth (Children and } & \text { Ask questions to ascertain use of tobacco products in multiple ways; use language and } \\ \text { Adolescents) } & \text { terminology that youth are familiar with. } \\ & \begin{array}{l}\text { Be aware of the natural history of tobacco use onset since there are important milestones from } \\ \text { 'first puff' to nicotine dependence that may signal transition to regular or daily smoking. Smoking } \\ \text { onset trajectories should be closely monitored, since intermittent smoking can quickly become } \\ \text { regular smoking. Ask, for example, about 'puffing' or 'trying' in addition to regular or daily use } \\ \text { (which indicate sustained smoking). }\end{array}\end{array}$

Aboriginal Peoples It should be emphasised that providers should recognise and distinguish between use of traditional (ceremonial/sacred) tobacco and misuse of commercial tobacco. Therefore, assessment and questions need to be conducted with care and respect for this difference.

Efforts should also be made to identify, engage and understand the range of resources available to provide appropriate referrals and connectivity to the Aboriginal community. For example, local First Nations communities, urban Aboriginal programme, Friendship Centres, etc.

Hospital-based Populations Approaches may differ for smokers admitted via emergency vs pre-admission, according to policies. In addition, some approaches may differ for patients who stop smoking for hospitalisations vs those patients who have a desire to quit while hospitalised.

Opportunity to discuss or prioritise the implementation of smoke-free policies in hospital settings can assist in establishing or supporting smoking cessation processes/programming. Examples can be drawn from institutions such as the Centre for Addiction and Mental Health

Mental Health and/or Other Consider that persons with mental illness and/or addiction(s) who smoke might need higher doses Addictions(s) of nicotine replacement therapy.

Clients' psychiatric symptoms throughout the quitting process should be monitored.

require as frequent updating given the robustness of the evidence to date for screening and counselling of tobacco use. In addition, the team constantly searches for updates in the source guidelines and the primary author (PS) is co-chairing the development of the updated Registered Nurses' Association of Ontario guidelines which was one of the source guidelines for CAN-ADPTT, due for release in 2017. We will use the criteria described by Cancer Care Ontario to be explicit as to whether the update validates or invalidates all or parts of the previous guideline, and/ or changes the strength of the evidence for each recommendation. ${ }^{44}$ Without ongoing funding, it is not feasible to keep the original GDG engaged in CAN-ADPTT activities. However, updates could be accomplished with limited funding and engagement via email/webcasts with a limited group of key stakeholders. Systematic reviews and meta-analyses, such as Cochrane reviews (tobacco. cochrane.org), could be appraised by the group and adapted to the Canadian context to provide updated recommendations. These could be posted online for public comment and emailed to network members for professional comments. Unfortunately, funding was not available for ongoing engagement of network members that could have been maintained with a listserv. In addition, new members would be required to replace members who leave or change the focus of their work. However, since funding has ended, ongoing dissemination and updates by other organisations has kept the guideline alive while we seek additional funding to conduct the updates.

The limitations of our process include the following. Although we tried using a wiki platform for dynamic discussion, use was minimal. We also were not able to tailor the information displayed on the website according to the audience reading the guidelines. There was no formal patient or public involvement in development of the guideline to prevent tokenism. Some HCPs and other target end-users who provided input may have been current or former smokers themselves or family members of people affected by smoking but we did not ask people to disclose this information to respect privacy. Only a minority of the large group of network members were 
engaged in the guideline development process. However, it is difficult to assess the success of our engagement efforts given the absence of similar projects with which to compare and the fact that the quality of the feedback received is difficult to quantify and evaluate. Lastly, we do not have data for patient-level outcomes across Canada to evaluate the impact of guideline implementation. Smoking prevalence rates continue to remain high but that is determined by more than clinical interventions per se.

This paper describes how to operationalise the ADAPTE framework in a highly resourced country where the culture differs across jurisdictions. We focused on respecting existing guidelines and focused on the factors that could increase adoption: wide stakeholder participation in the creation of the guideline; relevance to regional and local conditions by engaging funders and researchers in addition to clinical groups and use of technology and in-person meetings to develop and disseminate the guidelines. By placing them on the website and having a white board video by a famous influencer (Dr Mike Evans), we have made it accessible to patients as well (www.nicotinedependenceclinic.com/English/CANADAPTT/Pa ges/Home.aspx). Next steps for this initiative include continued knowledge translation efforts with a focus on guideline implementation as well as continued efforts to expand and strengthen the network. Opportunities to ensure sustainability of the guideline are being explored.

\section{CONCLUSION}

The CAN-ADAPTT initiative successfully used the ADAPTE process to create a Canadian guideline for smoking cessation. By engaging end-users to better understand needs, barriers and gaps at the outset, we were able to minimise the disconnect between knowledge creation and implementation. CAN-ADAPTT's work suggests that where high-quality guidelines already exist and funds are limited, methods to adapt existing guidelines to the local context are possible. Other groups undertaking a similar process should consider the value of engaging target end-users and deploying knowledge exchange brokers during guideline development.

\section{Author affiliations}

${ }^{1}$ Centre for Addiction and Mental Health, Addictions Program, Toronto, Ontario, Canada

${ }^{2}$ Department of Family and Community Medicine, University of Toronto, Toronto, Ontario, Canada

${ }^{3}$ Department of Psychiatry, University of Toronto, Toronto, Ontario, Canada ${ }^{4}$ Dalla Lana School of Public Health, University of Toronto, Toronto, Ontario, Canada ${ }^{5}$ Centre for Effective Practice, Toronto, Ontario, Canada

${ }^{6}$ Education, Centre for Addiction and Mental Health, Toronto, Ontario, Canada ${ }^{7}$ Institut National de Santé Publique du Québec, Montreal, Québec, Canada ${ }^{8}$ Department of Pharmacology and Toxicology, University of Toronto, Toronto, Ontario, Canada

Contributors PS was the principal investigator and provided overall supervision of the project at all stages. PS, KH, JR, KL-R, VC, MT, DK, RD, SH and LZ each made substantial contributions to the guideline development concept and process. $\mathrm{KH}$ wrote the initial draft of the manuscript. PS, KH, JR, KL-R, SS, VC, MT, DK, RD, SH and $L Z$ revised the manuscript critically for important intellectual content.

Funding Funding was provided by Health Canada (Drugs and Tobacco Initiatives Program).

Competing interests No pharmaceutical or tobacco industry funds were used or received in the development of these guidelines. During the course of the grant, PS received funding for ad hoc consulting for advisory boards, education and unrestricted research grants from manufacturers of smoking cessation medications including Pfizer, Johnson and Johnson, Nabi Pharmaceuticals via an arm's length Data Safety and Monitoring Board, Novartis and Biovail. PS also received consulting fees from manufacturers of anti-addiction medications including Prempharm, Lundbeck and Sanofi Synthélabo. No funds for entertainment were accepted. VC reports grants from Drugs and Tobacco Initiatives Program, Health Canada, during the conduct of the study. LZ reports grants from Global Research Awards for Nicotine Dependence, and a grant from Pfizer Canada, outside the submitted work. The remaining authors have nothing to disclose.

Provenance and peer review Not commissioned; externally peer reviewed. Data sharing statement № additional data are available.

Open Access This is an Open Access article distributed in accordance with the Creative Commons Attribution Non Commercial (CC BY-NC 4.0) license, which permits others to distribute, remix, adapt, build upon this work non-commercially, and license their derivative works on different terms, provided the original work is properly cited and the use is non-commercial. See: http://creativecommons.org/ licenses/by-nc/4.0/

C) Article author(s) (or their employer(s) unless otherwise stated in the text of the article) 2017. All rights reserved. No commercial use is permitted unless otherwise expressly granted.

\section{REFERENCES}

1. Solberg LI, Maciosek MV, Edwards NM, et al. Repeated tobacco-use screening and intervention in clinical practice: health impact and cost effectiveness. Am J Prev Med 2006;31:62-71.

2. Stead LF, Buitrago D, Preciado N, et al. Physician advice for smoking cessation. Cochrane Database Syst Rev 2013;31:CD000165.

3. Fiore M, Jaén C, Baker T, et al. Treating tobacco use and dependence: 2008 update. Rockville, MD: U.S. Department of Health and Human Services, 2008.

4. West R. The clinical significance of "small" effects of smoking cessation treatments. Addiction 2007;102:506-9.

5. Centers for Disease Control and Prevention (CDC). Smokingcessation advice from health-care providers-Canada, 2005. MMWR Morb Mortal Wkly Rep 2007;56:708-12.

6. Grol R, Buchan H. Clinical guidelines: what can we do to increase their use? Med J Aust 2006;185:301-2.

7. Graham ID, Beardall S, Carter AO, et al. The state of the science and art of practice guidelines development, dissemination and evaluation in Canada. J Eval Clin Pract 2003;9:195-202.

8. Woolf $\mathrm{S}$, Schünemann $\mathrm{HJ}$, Eccles MP, et al. Developing clinical practice guidelines: types of evidence and outcomes; values and economics, synthesis, grading, and presentation and deriving recommendations. Implement Sci 2012;7:61.

9. Eccles MP, Grimshaw JM, Shekelle P, et al. Developing clinical practice guidelines: target audiences, identifying topics for guidelines, guideline group composition and functioning and conflicts of interest. Implement Sci 2012;7:1-8.

10. Shekelle P, Woolf S, Grimshaw JM, et al. Developing clinical practice guidelines: reviewing, reporting, and publishing guidelines; updating guidelines; and the emerging issues of enhancing guideline implementability and accounting for comorbid conditions in guideline development. Implement Sci 2012;7:62.

11. World Health Organization. WHO framework convention on tobacco control. Geneva, Switzerland, 2003.

12. Ministry of Health. New Zealand smoking cessation guidelines. Wellington: Ministry of Health, 2007.

13. Stead LF, Perera R, Bullen C, et al. Nicotine replacement therapy for smoking cessation. Cochrane Database Syst Rev 2008;11:CD000146.

14. Stead LF, Bergson G, Lancaster T. Physician advice for smoking cessation. Cochrane Database Syst Rev 2008;2:CD000165.

15. Harrison MB, Legare F, Graham ID, et al. Adapting clinical practice guidelines to local context and assessing barriers to their use. Can Med Assoc J 2010;182:E78-E84. 
16. Graham R, Mancher M, Wolman DM, et al. Clinical practice guidelines we can trust. Washington, DC: National Academies Press, 2011.

17. Qaseem A, Forland F, Macbeth F, et al. Guidelines International Network: toward international standards for clinical practice guidelines. Ann Intern Med 2012;156:525-31.

18. Graham ID, Logan J, Harrison MB, et al. Lost in knowledge translation: time for a map? J Contin Educ Health Prof 2006;26:13-24.

19. The ADAPTE Collaboration. The ADAPTE process: resource toolkit for guideline adaptation. version 2.0, 2009.

20. AGREE Collaboration. Development and validation of an international appraisal instrument for assessing the quality of clinical practice guidelines: the AGREE project. Qual Saf Health Care 2003;12:18-23.

21. Straus SE, Tetroe J, Graham I, et al. Defining knowledge translation. CMAJ 2009;181:165-8.

22. Gagnon ML. Moving knowledge to action through dissemination and exchange. J Clin Epidemiol 2011;64:25-31.

23. Fervers B, Burgers JS, Haugh MC, et al. Adaptation of clinical guidelines: literature review and proposition for a framework and procedure. Int J Qual Health Care 2006;18:167-76.

24. Palda VA, Rogers J, Kapur A, et al. The Guidelines Advisory Committee: Ontario's approach to guideline appraisal and adoption. Toronto, Canada: Guidelines International Network Annual Conference, 2007.

25. CAN-ADAPTT. Canadian smoking cessation clinical practice guideline, 2011. https://www.nicotinedependenceclinic.com/ English/CANADAPTT/Documents/CAN-ADAPTT\%20Canadian\% 20Smoking\%20Cessation\%20Guideline_website.pdf (accessed 4 Oct 2016).

26. Guyatt G, Gutterman D, Baumann MH, et al. Grading strength of recommendations and quality of evidence in clinical guidelines: report from an american college of chest physicians task force. Chest 2006;129:174-81.

27. UpToDate. Grading guide. http://www.uptodate.com/home/gradingguide.

28. Guyatt GH, Oxman AD, Vist GE, et al. Rating quality of evidence and strength of recommendations: GRADE: an emerging consensus on rating quality of evidence and strength of recommendations. Br Med $J$ 2008;336:924-6.

29. Registered Nurses' Association of Ontario. Integrating smoking cessation into daily nursing practice (revised). Toronto, Canada: Registered Nurses' Association of Ontario, 2007.
30. Health Canada. First Nations \& Inuit Health. Tobacco. 2011 http:// www.hc-sc.gc.ca/fniah-spnia/substan/tobac-tabac/index-eng.php

31. Lasser K, Boyd JW, Woolhandler S, et al. Smoking and mental illness. JAMA 2000;284:2606-10.

32. Chen J, Millar WJ. Age of smoking initiation: implications for quitting Health Rep 1998;9:39-46.

33. Hawn MT, Houston TK, Campagna EJ, et al. The attributable risk of smoking on surgical complications. Ann Surg 2011;254:914-20.

34. Einarson A, Riordan S. Smoking in pregnancy and lactation: a review of risks and cessation strategies. Eur J Clin Pharmacol 2009;65:325-30.

35. Els C, Kunyk D, Selby P. Disease interrupted: tobacco reduction and cessation. Charleston, SC: CreateSpace, 2014.

36. Herie M, Connolly $\mathrm{H}$, Voci S, et al. Changing practitioner behavior and building capacity in tobacco cessation treatment: the TEACH project. Patient Educ Couns 2012;86:49-56.

37. Tobe SW, Stone JA, Walker KM, et al. Canadian cardiovascular harmonized national guidelines endeavour (C-CHANGE): 2014 update. CMAJ 2014;186:1299-305.

38. CAN-ADAPTT. Canadian smoking cessation clinical practice guideline. http://www.strokebestpractices.ca/wp-content/uploads/ 2012/04/CAN-ADAPTT2.pdf (accessed 13 Jul 2017).

39. Canadian Society of Respiratory Therapists. Catalogue of guidelines. http://www.csrt.com/catalogue-of-guidelines/ (accessed 13 Jul 2017).

40. CAN-ADAPTT summary statements. http://www.peelregion. ca/health/professionals/events/pdf/2013/canadaptt-summarystatements.pdf (accessed 13 Jul 2017).

41. Kredo T, Bernhardsson S, Machingaidze S, et al. Guide to clinical practice guidelines: the current state of play. Int J Qual Health Care 2016;28:122-8

42. Davis DA, Goldman JB, Palda VA. Canadian medical association handbook on clinical practice guidelines. Ottawa, Canada: Canadian Medical Association, 2007.

43. Vernooij RW, Sanabria AJ, Solà I, et al. Guidance for updating clinical practice guidelines: a systematic review of methodological handbooks. Implement Sci 2014;9:3.

44. Johnston ME, Brouwers MC, Browman GP. Keeping cancer guidelines current: results of a comprehensive prospective literature monitoring strategy for twenty clinical practice guidelines. Int $J$ Technol Assess Health Care 2003;19:646-55.

45. Priest N, Roseby R, Waters E, et al. Family and carer smoking control programmes for reducing children's exposure to environmental tobacco smoke. Cochrane Database Syst Rev 2008;4:CD001746. 